\title{
LOS RENDIMIENTOS A LA ESCOLARIDAD EN EL AREA METROPOLITANA DE MONTERREY
}

\author{
YVONNE STINSON ORTIZ
}

\section{INTRODUCCIÓN}

ESTE TRABAJO RESUME LOS RESULTADOS de un estudio, ${ }^{1}$ dedicado a probar hipótesis fundamentales de la teoría del capital humano y a suministrar estimaciones de las tasas marginales de rendimiento a la escolaridad; se basa en la información proveniente de la "encuesta continua de mano de obra" correspondiente al cuatrimestre enero-abril de 1978.

En la primera sección se presenta el marco teórico que considera la educación como inversión y se derivan de él seis hipótesis de trabajo, así como los modelos para ser utilizados en la prueba de las mismas. En la segunda, se exponen los resultados de dichas pruebas. $Y$ finalmente, en la tercera se presentan las conclusiones.

Dos de los resultados más importantes del estudio son la evidencia encontrada sobre: a) la relación positiva entre los niveles de escolaridad y las tasas marginales de rendimiento correspondientes; $b /$ la ausencia de diferencias significativas entre las tasas marginales de rendimiento a la escolaridad de hombres y mujeres. Con base en estos resultados se formulan las conclusiones del trabajo y las implicaciones de política económica correspondientes.

1 Stinson Ortiz, Yvonne, M.H. Los determinantes del ingreso laboral: el enfoque de rendimientos a la escolaridad. Tesis profesional, Universidad Autónoma de Nuevo León. 1979. 


\section{MODELO TEÓRICO}

Analizando el comportamiento del mercado de trabajo, con frecuencia se observa que, en igualdad de circunstancias, los individuos que poseen determinadas capacidades (ya sea innatas o adquiridas) son remunerados en forma diferente.

En el caso de las capacidades adquiridas, éstas quedan comprendidas en el concepto de capital humano. El estudio sobre la acumulación de capital humano se ha realizado utilizando como base las técnicas existentes para el análisis de la acumulación del capital físico; por lo tanto, se considera que durante su ciclo de vida el individuo se enfrenta a posibilidades de inversión, ${ }^{2}$ una de las cuales consiste en incrementar la calidad de su trabajo, y decide a favor de aquellas de las que espera mayor rentabilidad. Como lo señalé anteriormente, existen dos formas en las cuales se ha estimado la rentabilidad de las inversiones en educación; una que utiliza los criterios de valor presente neto y de tasa interna de rendimiento, parámetros que son obtenidos a partir de estimaciones de beneficios y costos para cada uno de los diferentes niveles educativos; $y$ otra que se basa en estimaciones de la tasa de rendimiento externo para cada uno de los diferentes niveles educativos. Estas estimaciones se obtienen a partir de modelos similogarítmicos de regresión múltiple.

En el presente trabajo, concentraremos la atención sobre escolaridad y experiencia como formas de acumulación de capital humano. Con relación a la escolaridad, la primera hipótesis que se plantea es que a medida que sea mayor la cantidad de tiempo invertido, y por lo tanto los costos totales, mayor será la cantidad y calidad de las capacidades invertidas, así como el desplazamiento esperado en el perfil de ingresos, es decir, se plantea una relación positiva entre escolaridad e ingresos provenientes del trabajo; de manera semejante se deriva la segunda hipótesis, la cual plantea una relación positiva entre experiencia e ingresos provenientes del trabajo. En resumen, estableciendo el supuesto de continuidad en las variables experiencia $(T)$ y escolaridad $(S)$, se plantea una relación funcional:

$$
\text { (1) } Y=Y(S, T) \text {, donde } Y_{S^{\prime}}^{\prime}>0 \text { y } Y_{T}^{\prime}>0
$$

Cuando se tienen en cuenta las capacidades no ac uiridas, éstas se engloban en el concepto habilidad $(A)$, la cual es medida con $\mathrm{f}$ :uencia por medio del cociente intelectual, dando lugar a la transformación de . expresión (1) en:

(2) $Y=Y(A, S, T)$, donde $Y_{A}^{\prime}>0, Y_{S}^{\prime \prime} ;$ y $Y_{T}^{\prime}>0$

El estudio de la relación empírica entre ingreso y escolaridad se puede llevar a cabo empleando múltiples especificaciones del modelo ${ }^{3}$ sin embargo, dado que

2 Jack Hischleifer, "On the Optimal Theory of Investment". Journal of Political Econo$m y$, Vol. 66, Núm. 4, agosto de 1958, pp. 329-332, señala que el individuo toma simultáneamente sus decisiones de ahorro e inversión. Cuando los mercados de capitales son perfectos, dichas decisiones son independientes entre sí.

${ }^{3}$ James Heckman y Solomon Polancheck, "The Funcional Form of the Income School- 
nuestro interés consiste en la obtención de estimaciones de las tasas de rendimiento sobre la escolaridad, nos concentraremos en aquellas que satisfacen los detalles que derivaremos a continuación.

Iniciaremos la derivación de nuestro modelo ${ }^{4}$ estableciendo los supuestos siguientes: 1) que los únicos costos privados de capacitación consisten en los salarios que se dejan de percibir;2) que la especialización es completa en las actividades de capacitación, y por lo tanto, mientras dura no se recibe salario; y 3) que existe homogeneidad en la habilidad de las personas. A partir de ellos, un individuo que en el presente período desea dedicarse a obtener el nivel $J$ de escolaridad, tendria que incurrir en costos totales iguales al salario que dejaría de percibir (el cual correspondería al nivel de escolaridad $J-1$ ), es decir, que en la derivación se ignoran los costos directos privados de la educación por lo que la relación entre los costos totales y el ingreso dejado de percibir es igual a la unidad. De acuerdo con esto, una persona que ve la escolaridad como una inversión de la cual se obtienen rendimientos positivos, encontraría que, de decidirse a alcanzar el primer nivel de escolaridad su ingreso se transformaría en:

(3) $Y_{1}=Y_{0}+r_{1} Y_{0}$;

donde $r_{1}$ es el incremento porcentual que obtendría en su nivel de ingreso, y $Y_{0}$ es el costo en que tendría que incurrir a fin de cambiar su perfil de ingresos.

En el caso en que la decisión se refiriese al segundo año de escolaridad, encontraría que a partir del próximo período, podría comenzar a obtener un ingreso igual a:

(4) $Y_{2}=Y_{1}\left(1+r_{2}\right)$

donde $Y_{1}$ y $r_{2}$ tienen una interpretación semejante a la indicada en la relación anterior.

Como se puede observar, la expresión (4), puede reformularse como:

(4a) $Y_{2}=Y_{0}\left(1+r_{1}\right)\left(1+r_{2}\right)$

y que basándonos en ella podemos expresar el ingreso correspondiente a $S$ affos de escolaridad como:

$$
\text { (5) } Y_{S}=Y_{0} \prod_{j=1}^{S}\left(1+r_{j}\right)
$$

donde $\pi$ es el símbolo de la multiplicación, $Y_{S}$ es el nivel de ingreso proveniente del trabajo que corresponde a $S$ años de escolaridad, $Y_{0}$ es el ingreso para quien no tiene escolaridad, y $r_{i}$ es la tasa de rendimiento sobre el nivel de escolaridad $j$.

ing Relationship". Joumal of the American Statistical Association, vol. 69, núm. 346, junio de 1974. 1974 .

${ }^{4}$ Barry Chiswick, Income Inequality. Cap. III. Columbia University Press, Nueva York, 
Los supuestos de que no hay costos de capacitación y de que no se perciben salarios durante la inversión pueden parecer poco realistas; la existencia de costos directos en forma de colegiatura, material escolar y otros gastos empleados por la escolaridad llevan a que la razón $\left(K_{j}\right)$ entre los costos totales y los ingresos perdidos empleados por el $J$ nivel de escolaridad, tenga un valor mayor a la unidad, y que por lo tanto afecte las estimaciones de las tasas de retorno.

Considerando un $K_{j}>1$, se encuentra que los costos totales para alcanzar el nivel $J$ implican unos costos totales (directos más ingreso dejado de percibir), igual a $K_{j} \times Y_{j-1}$; siendo $Y_{j}$ igual a $Y_{j-1}$ más el rendimiento obtenido sobre los costos totales en que se incurrió al alcanzar el nivel de escolaridad en cuestión, encontramos que:

$$
\begin{aligned}
& Y_{j}=Y_{j-1}+r_{j}(C T)_{j} \\
& Y_{j}=Y_{j-1}+r_{j}\left(K_{j} Y_{j-1}\right) \\
& \quad \text { (6) } Y_{j}=Y_{j-1}+r_{j}^{*} Y_{j-1}
\end{aligned}
$$

donde $r_{j}^{*}$ es una tasa de retorno ajustada cuando $J=1$, es decir que la decisión se refiere al primer nivel de escolaridad.

(6a) $Y_{1}=Y_{o}+r_{1}\left(K_{1} Y_{o}\right)=Y_{o}\left(1+r_{1} K_{1}\right)=Y_{o}\left(1+r_{1}^{*}\right)$;

utilizando la generalización expresada por la fórmula (5), encontramos que:

(7) $Y_{s}=Y_{o} \underset{j=1}{s}\left(1+r_{j}^{*}\right)$

aplicando $1_{n}$ se obtiene:

(8) $1_{n} Y_{s}=1_{n} Y_{o}+1_{n} \prod_{j=1}^{s}\left(1+r_{j}^{*}\right)$

si el $1_{n}(1+r) \approx r$ (para $r$ pequeños) por lo que:

(9) $1_{n} Y_{s}=1_{n} Y_{o}+\sum_{j=1}^{s} r_{j}$

(9a) $1_{n} Y_{s}=1_{n} Y_{o}+r^{*} S+U$

Analizando la expresión ( $9 a$ ), nos damos cuenta que esta especificación del modelo puede ayudarnos a probar la relación funcional entre ingreso y escolaridad, pues en ella el coeficiente de $S$ resulta ser una estimación de la tasa de rendimiento a la educación (en forma de escolaridad). Esto es porque si el ingreso pasado es el único costo considerado de un año adicional de escolaridad, y si el incremento en el logaritmo del ingreso certifica a este año adicional de escolaridad (r) ser constante y perpetuo, entonces $r$ se aproxima a $(\alpha Y / \alpha s) / Y$, y tiene la 
interpretación de la "tasa de rendimiento de la inversión en educación"; embargo, la igualdad de las tasas de rendimiento para cada uno de los niveles de escolaridad puede parecer un supuesto sumamente restrictivo. Por ello, otra formulación podría ser que las tasas de rendimiento se encuentran relacionadas positivamente con el nivel de escolaridad, lo cual reflejaría una escasez relativa de trabajo calificado o viceversa, pudiendo ser representada esta relación por la expresión:

(10) $r_{i}=\alpha_{0}+\alpha_{1} S_{1}$

donde un valor positivo para $\alpha_{1}$ implicaría el caso en que las tasas de rendimiento se correlacionaran positivamente con el nivel de escolaridad. Sustituyendo la expresión (10) en la (8) obtendremos:

$$
\begin{aligned}
& 1_{n} Y_{s}=1_{n} Y_{o}+1_{n} \prod_{i=1}^{s}\left(1+r_{i}\right) \\
& 1_{n} Y_{s}=1_{n} Y_{o}+\sum_{i=1}^{s} r_{i} \\
& 1_{n} Y_{s}=1_{n} Y_{o}+\sum_{i=1}^{s}\left(\alpha_{o}+\alpha_{i}\right) \\
& \text { (11) } 1_{n} Y_{s}=1_{n} Y_{o}+\beta_{o} S+\beta_{1} s^{2}
\end{aligned}
$$

analizando la expresión (11) nos damos cuenta de que, conociendo los parámetros de la educación (11), podemos estimar las tasas marginales de rendimiento a la educación $\left(r_{s}\right)$, ya que:

$$
\text { (12) } r_{s}=\frac{\delta 1_{n} Y_{s}}{\delta_{S}}=\beta_{o}+2 \beta_{1} S
$$

Asimismo, estas estimaciones de las tasas marginales de rendimiento a la educación nos permiten probar la hipótesis que plantea la existencia de una escasez relativa de trabajo calificado, entendiendo por éste, el que requiere mayores niveles de escolaridad. La prueba de esta tercera hipótesis se puede llevar a cabo observando si $\beta$ tiene un valor positivo que difiera significativamente de cero.

Como se señala en la expresión (2), existen otros factores que afectan el ingreso de los individuos y que quedan comprendidos dentro del concepto de capital, éstos son la habilidad y la experiencia (nos abstraemos de los programas

5 Zvi. Griliches, "Lstimating the Retunms to Schooling: Some Econometric Problems". Econometrica, vol. 45, núm. 1., enero de 1977. 
de adiestramiento en el trabajo). La relación funcional entre ingreso y experiencia $(T)$, o entre ingreso y habilidad puede ser planteada de una manera sencilla, en la expresión siguiente:

$$
\text { (13) } Y_{T}=Y_{o}{\underset{i=1}{T}}_{i=1}^{T}\left(1+\theta_{i}\right)
$$

la cual puede ser transformada en:

$$
\text { (14) } 1_{n} Y_{T}=1_{n} Y_{o}+\sum_{i=1}^{T} 1_{n}\left(1+\theta_{i}\right)
$$

En el caso especial en que $\theta_{1}=\theta_{2}=\theta_{3}=\ldots \ldots \ldots=\theta_{T}$, obtendremos la expresión:

$$
\text { (15) } 1_{n} Y_{T}=1_{n} Y_{o}+\theta T_{1}
$$

la cual resulta ser semejante a ( $9 a)$, excepto porque en este caso $\theta$ no representa una estimación sobre la tasa de rendimiento a la experiencia, ya que mientras que ésta se obtiene no existe sacrificio de ingresos. Una formulación semejante a $(9 a)$ y $(15)$ puede ser encontrada para la habilidad $(A)$, por lo que considerando conjuntamente estas expresiones, se obtiene:

$$
\text { (16) } 1_{n} Y_{T}=1_{n} Y_{o}+\beta_{1} S+\beta_{2} T+\beta_{3} A \text {; }
$$

donde $\beta_{1}$ es nuestro estimador de la tasa marginal de rendimiento a la escolaridad. Estudios realizados en otros países ${ }^{6}$ muestran una fuerte correlación entre las variables $S, T$ y $A$; lo cual nos hace pensar que (15) es una especificación más adecuada que (9a) para la estimación de las mencionadas tasas de rendimiento, ya que evita los sesgos positivos que surgieran por la incorrecta especificación del modelo. ${ }^{7}$

Como lo señalábamos anteriormente, especificaciones del modelo semejante a (16) pueden estar restringiendo significativamente el valor de los estimadores de la tasa de rendimiento a la escolaridad. Con la ecuación (11) podemos llegar a una nueva especificación del modelo. De manera semejante, inspirados en las proposiciones de la hipótesis del ciclo de vida de la función consumo, en lo que respecta a la forma de los perfiles de ingresos, podemos haver consideraciones sobre la contribución de la experiencia planteando que,

$$
\text { (17) } \theta=r_{o}+r_{1} T
$$

6 Zvi Griliches y William Mason, "Education, Income and Ability". Journal of Political Economy, vol. 80, núm. 3, parte II, mayo-junio de 1972.

7 Johnston, J., Econometric Methods, 2a. edición, McGraw Hill Kogakusha, Ltd., Tokio, 1972. 
lo cual permite observar si el perfil de ingresos, correspondiente a determinado nivel de escoiaridad, se conforma con la hipótesis mencionada i.e., en la forma que nos indica la gráfica 1.

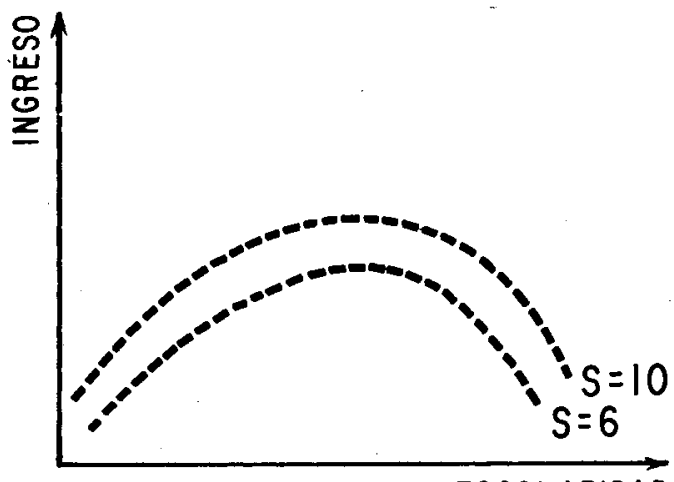

ESCOLARIDAD

Teniendo en cuenta dichas sugerencias derivamos la ecuación:

(18) $1_{n} Y=1_{n} Y_{0}+B_{1} S+B_{2} S+B_{3} T+B_{4} T^{2}+B_{5} A$,

de donde nuestra tercera y cuarta hipótesis de trabajo plantean que:

$$
B_{2}>0, B_{3}>0 \text { y } B_{4}<0
$$

Estudiando el comportamiento de las tasas de rendimiento a la educación en Estados Unidos, a través del tiempo, Finis Welch ${ }^{8}$ encontró que dichos rendimientos se veían incrementados durante situaciones de "desequilibrio", es decir, durante situaciones caracterizadas por cambios continuos. Para Finis Welch y Wallace Hauffmann, ${ }^{9}$ la educación proporciona al individuo capacidad para adquirir y codificar información a menores costos, lo cual le permite un mejor desempeño en el trabajo y por lo tanto mayor rendimiento a su inversión. El argumento de Hauffman va aun más allá: establece que ante cambios únicos en el estado de cosas, la educación permite ventajas temporales, y que al fluir la información, los no educados aprenden mediante la experiencia y esto origina la reducción de los rendimientos recibidos por los no educados.

De esta manera, la experiencia y la escolaridad son opciones para adquirir conocimientos y capacidades. Ante este tipo de argumentos surge la inquietud por considerar los tipos de ocupación como variables explicativas de los ingresos provenientes del trabajo, lo cual da lugar a que la ecuación (18) se transforme en:

${ }^{8}$ F. Walch, "Black and White Rates of Return to Schooling". American Economic Review, vol. 63, núm. 5, diciembre de 1973.

9 "Decision Making: The Role of Education", American Joumal of Agricultural Economics, febrero de 1974, pp. 81-97. 
(19) $1_{n} Y=1_{n} Y_{o}+\beta_{1} S+\beta_{2} S^{2}+\beta_{3} T+\beta_{4} T^{2}+\beta_{5} A+\sum_{i=6}^{n} \beta_{i} D_{i}$

donde $D_{i}$ es una variable dummy que permite considerar los diferentes tipos de ocupación. En la medida en que estos tipos de ocupación influyan sobre el ingreso y se encuentren fuertemente correlacionados con los niveles de escolaridad, la ecuación (19) resulta más adecuada para la estimación de los rendimientos a la educación pues evita los sesgos provenientes de la omisión de variables.

Se puede pensar también que las situaciones dinámicas o de "desequilibrio", más que con el tipo de ocupación se encuentran fuertemente relacionadas con los diferentes sectores de la actividad económica; por lo tanto el sector de actividad económica es una variable importante en la explicación de los diferenciales de ingreso reales. Este tipo de razonamiento da lugar a la derivación de un modelo como el representado por la ecuación (19), donde las variables dummy permiten considerar los diferentes sectores de la actividad económica. La consideración de estos sectores surge ante el deseo de reducir los sesgos en la estimación de las tasas marginales de rendimiento ya que la ausencia de información sobre cuestiones genéticas, ambiente familiar (desempeño profesional de padres y hermanos), selección de muestra, etc., introduce sesgos cuyo signo final resulta difícil de determinar.

Una nota final sobre nuestros modelos para la estimación de las tasas de rendimiento a la educación escolar se relaciona con la posibilidad de que exista discriminación por sexo en el mercado de trabajo; lo cual originaría diferencias de rendimientos de la educación, así como de contribución de la experiencia, entre hombres y mujeres. Al respecto, la quinta hipótesis nula planteará que tal discriminación no existe, $y$, por lo tanto, que no existe una diferencia significativa entre los coeficientes de ambos grupos.

De esta manera, quedan establecidas las ecuaciones (16), (18) y (19), como las bases para la estimación de los rendimientos a los diferentes niveles de escolaridad; sin embargo, se explorará también cuáles son los efectos sobre las estimaciones de un modelo semejante a (16), cuando se introducen los sectores o el tipo de actividad como variables explicativas del nivel de ingreso.

\section{APLICACIÓN PARA EL ÁREA METROPOLITANA DE MONTERREY: MODELOS ECONOMÉTRICOS}

En la sección anterior se plantearon diversas hipótesis sobre la relación educacióningreso; asimismo, se derivaron los modelos econométricos que permiten probarlas y que pueden ser utilizados para obtener estimaciones de las tasas marginales y promedio de rendimiento a la escolaridad. El objeto de este apartado es mostrar los resultados obtenidos en la prueba de dicha hipótesis cuando fue utilizada la información proveniente de la "Encuesta continua de mano de obra" correspondiente al primer trimestre de 1978. 


\section{Información}

En el estado de Nuevo León, esta encuesta es levantada por la Secretaría de Programación y Presupuesto y la Dirección General de Estadística del gobierno del Estado, a través de ella se obtiene información trimestral sobre 900 familias.

La "Encuesta continua de mano de obra" consiste en el levantamiento de una serie de cuestionarios en períodos trimestrales, que tienen como objetivo principal "obtener información sobre la oferta, que constituye uno de los componentes del mercado de trabajo a nivel nacional y regional". ${ }^{10}$ En ella, la "unidad de selección es el hogar y sus componentes";1 y el tipo de muestreo utilizado es aleatorio y de etapas múltiples; en él, "las unidades de muestreo previo a la selección de los hogares son áreas geográficas perfectamente delimitadas, seleccionadas, con probabilidad proporcional en términos de población". 12

De la información proveniente de esta encuesta se seleccionó aquella que se relaciona con el área metropolitana de Monterrey, y que se refiere a los miembros del hogar que quedan clasificados como integrantes de la población económicamente activa. "Esta población está constituida por las personas de 12 años y más que en el período de referencia se encontraban ocupados, así como por los que tenían una situación de desocupación abierta". ${ }^{13}$

La información proporcionada por esta encuesta sobre el ingreso proveniente del trabajo, la edad, la escolaridad y otras características de los individuos que forman parte de la población económicamente activa, permite estudiar la relación entre educación e ingresos y ob tener como resultado, en el caso especial en que la atención se concentra en una relación funcional de tipo semilogarítmico, estimaciones de las tasas de retorno sociales a la educación que se recibe en forma de escolaridad. La obtención de tasas de tipo social responde al hecho de que la mencionada encuesta reporta el ingreso de los individuos antes de que se deduzca el impuesto sobre la renta, el cual resulta una aproximación de la contribución que hace el trabajo del individuo al producto nacional y por lo tanto al "bienestar social". Cuando el interés se refiere a las tasas de retorno privadas, el concepto relevante es el ingreso después del pago de dicho impuesto, o sea las percepciones netas que recibe el individuo.

\section{Resultados}

Son seis los modelos que se utilizan para las estimaciones mencionadas y seis las hipótesis de trabajo básicas que se presentan con estas estimaciones, estas hipótesis son las siguientes:

10 Secretaría de Programación y Presupuesto, Encuesta continua de mano de obra, vol. II, núm. 4, septiembre-diciembre de 1978, p. 16.
11 Ibid., p. 15.
12 Ibid., p. 18.
13 Ibid., p. 25. 
1. Existe en promedio una relación positiva entre escolaridad $(S)$ e ingreso proveniente dei trabajo $(Y)$.

2. $(S)$ y $(T)$ son complementarios entre sí.

3. Los retornos marginales a la educación son independientes de los niveles de escolaridad.

4. El perfil de ingresos provenientes del trabajo del individuo se conforma con lo señalado respecto a la hipótesis del ciclo de vida de la función consumo; por lo tanto, existe un nivel de experiencia $\left(T^{*}\right)$ en el que este ingreso llega a un máxim̧c.

5. Existe discriminación por sexo en el mercado de trabajo, lo cual se manifiesta por diferencias significativas en las tasas de retorno a la escolaridad entre hombres y mujeres.

6. En promedio, existe una relación positiva entre experiencias $(T)$ e ingreso proveniente del trabajo $(Y)$.

Para la estimación de los parámetros de cada uno de los modelos se utilizaron, en el procesamiento de la información, las subrutinas del paquete estadístico SPSS que se encuentra en el Centro Electrónico de Cálculo de la UANL. En el trabajo original, ${ }^{14}$ estas estimaciones se obtuvieron para cada uno de los trimestres de 1978; desafortunadamente existieron problemas de computación que evitaron se agregase la información de todos los trimestres y que se obtuvieran estimaciones a partir de ello, impidiendo con esto la realización de pruebas conjuntas de $F$ para probar que existían diferencias significativas en las estimaciones obtenidas para cada uno de los trimestres.

La consideración de estas hipótesis se realiza mediante la utilización de seis modelos. La prueba de los mismos se realiza con una cola y un error del $5 \%$.

\section{A. Modelo I. Ingreso como función lineal de la escolaridad, la experiencia y la ocupación,}

El modelo se encuentra representado por:

$$
\text { I. } 1_{n} Y_{i}=\alpha_{o}+\alpha_{1} S_{i}+\alpha_{2} D_{2}+\alpha_{3} T_{i}+U_{i}
$$

Debido a que contamos con información de corte transversal, el subíndice $i$ indica que nos referimos a las características del "iésimo individuo".

En el primero de los modelos intervienen como argumentos las variables escolaridad $(S)$ y experiencia $(T)$, las cuales se encuentran medidas en años completos y una variable ficticia $\left(D_{2}\right)$ que se utiliza para captar el estado de la ocupación, es decir, si la persona se encuentra ocupada $\left(D_{2}=1\right)$ o desocupada $\left(D_{2}=0\right)$. En lo que respecta a la experiencia, ésta se estima restando a la edad $(A)$ los años de escolaridad más seis, o dicho de manera diferente, $T=A-S-6$.

Analizando los parámetros estimados, se puede observar (cuadro I) que las variables $S$ y $T$ tienen signos positivos en cada una de las regresiones, conformán-

14 Véase la nota 1. 
dose, por lo tanto, con las deducciones provenientes del marco teórico que se expuso en la sección anterior. Al dirigir la atención hacia los coeficientes calculados de $t$, se observa que éstos tienen valores que difieren fuertemente de los valores de tablas que corresponden a un error del $5 \%$. Por lo tanto, las hipótesis (1) y (6) quedan probadas y con ello se encuentra evidencia sobre la relación positiva entre la escolaridad y la experiencia con los ingresos provenientes del trabajo y, por lo tanto, sobre el efecto de la escolaridad sobre los perfiles de ingreso. Como se señaló en la sección anterior, el coeficiente de la variante escolaridad tiene una importancia especial, pues resulta una estimación de la tasa promedio de retorno a la educación. Este coeficiente tiene un valor aproximado del 12 por ciento.

Estas estimaciones sobre el retorno promedio de la educación resultan ser significativamente menores a las estimaciones obtenidas por Martin Carnoy ${ }^{15}$ para las áreas metropolitanas de Monterrey, Guadalajara y México. Uná explicación de dichas diferencias puede ser que el estudio de Carnoy se dirige a la población económicamente activa masculina, que se encontraba ocupada, en tanto que los resultados que se han mencionado para el caso de Monterrey se refieren al total de la población económicamente activa. Este comentario resulta importante únicamente en la medida en que existan diferencias significativas entre el valor de los parámetros de este modelo para los hombres con respecto a las mujeres; es decir, en la inedida en que en el mercado de trabajo exista algún indicio de discriminación por sexo.

Tratando de considerar la hipótesis nula relacionada a la existencia de discriminación por sexo en el mercado de trabajo, se procedió a distinguir entre la población económicarnente activa masculina y femenina y a estimar los parámetros de este modelo para cada uno de estos grupos (cuadro 1). La hipótesis fue probada planteando la existencia de diferencias significativas entre los valores estimados de los parámetros para cada uno de estos grupos. Mediante el empleo de la distribución $F$ de Snedecor, ésta fue aceptada.

Analizando las estimaciones, se pudo detectar la razón de las diferencias en los coeficientes estimados para ambos grupos. En una primera etapa, el interés se concentró sobre la variable escolaridad, pues de existir diferencias significativas entre los rendimientos a la educación para ambos grupos, esto sería reflejo de la presencia de discriminación por sexo en el mercado de trabajo; utilizando la distribución $t$ de Student, se encontró que no existen diferencias significativas entre los rendimientos a la escolaridad entre hombres y mujeres.

De acuerdo con este resultado, encontramos que las estimaciones obtenidas por Carnoy para 1963 resultan significativamente mayores que las obtenidas en el presente estudio. Sin embargo, cabe hacer la aclaración de que la información utilizada por Carnoy proviene de una muestra no aleatoria; resultando por lo tanto imposible hacer las inferencias de las estimaciones de su muestra para la población; esto no sucede con las estimaciones del presente estudio, en el cual se utiliza una selección aleatoria de las unidades encuestadas.

15 Martin Camoy, The Cost and Return to Schooling in Mexico: A Case Study. Tesis de doctorado, Universidad de Chicago, 1964. 


\section{Cuadro 1}

Resultados de la regresión del logaritmo natural de ingreso del trabajo con escolaridad y experiencia para los hombres del área urbana de México, 1963

\begin{tabular}{|c|c|c|c|c|c|c|c|}
\hline \multirow{3}{*}{ Edad de los hombres } & \multicolumn{3}{|c|}{ Resumen de Estadisticas } & \multicolumn{4}{|c|}{ Regresiones } \\
\hline & $S D(\ln E)$ & $50(s)$ & $A V(1 n E)$ & $r$ & $b_{1} \quad b_{2}$ & $\operatorname{Var}(u)$ & $R^{2}$ \\
\hline & & (2) & (3) & (4) & (5) $(6)$ & (7) & (8) \\
\hline Todas las edades a & .89 & 3.98 & 6.94 & .14 & - & .46 & .42 \\
\hline & & & 6.57 & & & & \\
\hline 25 de edad ${ }^{\prime \prime}$ & .79 & 4.29 & 7.25 & .14 & $=$ & - & - \\
\hline \multicolumn{8}{|l|}{ Todas las } \\
\hline \multirow[t]{2}{*}{ edades } & .89 & 3.98 & 6.94 & .18 & $10-.0013$ & 3.22 & .73 \\
\hline & & & 6.57 & $(.002)$ & $(.002)$ & $(.0000)$ & \\
\hline
\end{tabular}

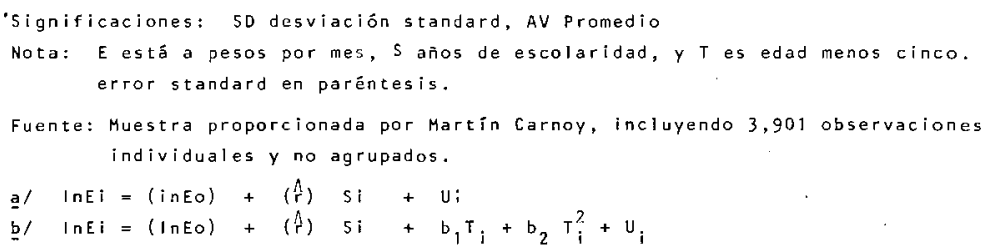

En el caso de la variable experiencia, los valores de sus coeficientes, para la población masculina, son positivos y difieren significativamente de cero. Ello muestra la relación positiva entre experiencia e ingresos provenientes del trabajo. Esto no es el caso del grupo de las mujeres, donde el coeficiente de esta variable no resulta significativamente diferente a cero. De esta manera encontramos una de las diferencias entre los grupos de coeficientes, de que se hablaba con anterioridad.

Con este primer modelo se pudo captar la relación positiva entre ingresos y escolaridad, obtenerse una estimación sobre los rendimientos a la educación, detectar discriminación por sexo en el mercado de trabajo, y encontrar que ésta no afecta los rendimientos promedio a la escolaridad; sin embargo, existe cierta preocupación por la posibilidad de que las variables que hemos omitido en la explicación de la varianza de $y$ se encuentren relacionadas con la escolaridad, y por lo tanto que se introduzcan mayores sesgos en las estimaciones del rendimiento a la educación.

\section{B. Modelo II. Escolaridad, experiencia y sectores de la actividad económica.}

En un segundo modelo sobre los ingresos del trabajo, podemos incorporar los sectores en los que se encuentra ocupado el individuo.

II. $1_{n} Y_{i}=\alpha_{o}+\alpha_{1} S_{i}+\alpha_{2} T_{i}+\alpha_{3} D_{2 i}+\alpha_{4} D_{4 i}+\alpha_{5} D_{5 i}+$ $\ldots:+\alpha_{13} D_{13 i}+U_{i}$ 
en donde $D_{4 i} \ldots, D_{13 i}$, son variables dummy que permiten considerar los sectores de la actividad económica; correspondiendo a los sectores industria extractiva $\left(D_{4}\right)$, industria manufacturera $\left(D_{5}\right)$, industria de la construcción $\left(D_{6}\right)$, electricidad y gas $\left(D_{7}\right)$, comercio $\left(D_{8}\right)$, transporte $\left(D_{9}\right)$, servicios financieros $\left(D_{10}\right)$, servicios médicos $\left(D_{13}\right)$, respectivamente.

Una vez estimados los parámetros de este modelo con la información sobre la población económicamente activa total, masculina y femenina (cuadro 2), la atención se concentró sobre los coeficientes de las variables escolaridad y experiencia. El coeficiente de la primera variable tuvo un valor de $10 \%$, positivo y significativamente diferente de cero para cada uno de los grupos. En lo que respecta a la variable experiencia, el coeficiente estimado tiene un valor positivo que difiere significativamente de cero, para el caso general y el grupo masculino; sin embargo, en el caso de las mujeres se observó un patrón semejante al del modelo anterior, es decir, que el coeficiente estimado de la variable no difiere significativamente de cero.

Como se puede observar en los dos modelos que han sido analizados, existe evidencia sobre la relación positiva entre escolaridad e ingreso proveniente del trabajo; sin embargo, en el caso de la relación entre ingreso y experiencia, la evidencia muestra que sólo se puede probar la hipótesis para el caso general o para el del grupo masculino. Por otra parte, se puede observar que la inclusión de los sectores de actividad económica, como el lugar de trabajo, incrementa significativamente los coeficientes de la escolaridad y experiencia, que son los que interesan en el presente estudio.

\section{Modelo III. Escolaridad, experiencia, sectores de actividad económica y horas trabajadas}

Un tercer modelo se puede plantear si incluimos el número de horas trabajadas por semana $(H T)$. El modelo se ve transformado en:

$$
\text { III. } \begin{aligned}
1_{n} Y_{i}= & \alpha_{o}+\alpha_{1} S_{i}+\alpha_{2} T_{i}+\alpha_{3} D_{2 i}+\alpha_{4} D_{4 i}+\ldots \ldots \ldots+ \\
& \alpha_{13} D_{13 i}+\alpha_{14} H T_{i}+U_{i}
\end{aligned}
$$

La variable número de horas trabajadas por semana $(H T)$ puede encontrarse correlacionada con el nivel de escolaridad y, por lo tanto, su omisión puede ocasionar sesgos en las estimaciones de los rendimientos a la educación.

Al hacer las estimaciones de los parámetros de este modelo (cuadro 3), para los grupos masculino y femenino, se encontró que el coeficiente de la variable $H T$ tiene un valor positivo que difiere significativamente de cero. Asimismo, que los coeficientes de las variables $S$ y $T$ no se vieron alterados significativamente con la introducción de la variable $H T$, por lo que se observa en las estimaciones un patrón semejante al del modelo anterior, obteniéndose una estimación del rendimiento social promedio a la educación que difiere significativamente, del $12 \%$ (a un nivel de confianza del 95\%).

Una vez observado que las estimaciones del rendimiento social promedio de la 
escolaridad, obtenidas bajo los tres modelos mencionados anteriormente, no difieren significativamente entre sí, surge la conveniencia de estimar los rendimientos sociales sobre esta inversión y de probar la hipótesis de que éstos se encuentran estrechamente relacionados con los niveles de escolaridad. Un modelo adecuado para trabajar sobre esta línea es el siguiente:

D. Modelo IV. Escolaridad, experiencia, ocupación y su interrelación (función cuadrática)

IV. $1_{n} Y_{i}=\alpha_{o}+\alpha_{1} S_{i}+\alpha_{2} S_{i}^{2}+\alpha_{3} T_{i}+\alpha_{4} T_{i}^{2}+$

$$
\ldots \alpha_{5} S_{i} T_{i}+\alpha_{6} D_{2 i}+U_{i}
$$

Como se explicó en el capítulo anterior, al utilizarse este modelo la tasa de retorno social sobre la escolaridad está dada por:

$$
\text { i) } r_{s}=\frac{\delta 1_{n} Y_{i}}{\delta S_{i}}=\alpha_{1}+2 \alpha_{2} S_{i}+\alpha_{5} T_{i}
$$

por lo que, de tener $\alpha_{2}$ un valor significativamente diferente de cero, implica que $r_{s}$ se encuentra estrechamente relacionado con el vałor de $S_{i}$ por medio de la función $(i)$.

Por otra parte, al incorporar la variable $T^{2}$ al modelo es posible probar la hipótesis que establece que el perfil de ingreso de la población económicamente activa del área metropolitana de Monterrey, se conforma con lo que propone la hipótesis del ciclo de vida. Como se estableció con anterioridad, ${ }^{16}$ una forma estadística de probar esta hipótesis consiste en postular que:

$$
\hat{\alpha}_{4}<\begin{array}{llllll}
0 & \text { y } & \alpha_{3} & >0
\end{array}
$$

Finalmente, es importante señalar que la incorporación de la variable $S T$, de interacción, permite estudiar si las capacidades adquiridas por la escolaridad y la experiencia son complementarias, se esperaría un signo positivo en el coeficiente de esta variable y el signo contrario para el caso de sustitución entre dichas capacidades.

Al analizar las estimaciones correspondientes a este modelo, se puede observar que para la población económicamente activa, los coeficientes estimados de la variable $S_{1}$ no difieren significativamente de cero (con un nivel de confianza del $95 \%$ ), en tanto que el de la variable $S^{2}$ tiene un valor positivo que difiere significaticamente de cero. De esta manera, encontramos evidencia para la tercera hipótesis de trabajo, ${ }^{17}$ la cual plantea que los rendimientos marginales de la educación eran independientes del nivel de escolaridad; rechazamos esta hipóte-

16 Véase p. 15, hipótesis 4.

17 Véase p. 13, hipótesis 3. 
sis nula al encontrar evidencia de que el coeficiente de la variable $S^{2}$ difiere significativamente de cero.

Tratando de encontrar evidencia sobre la sexta hipótesis de trabajo, ${ }^{17}$ la cual establece que las variables escolaridad y experiencia son complementarias, se concentró la atención sobre el coeficiente estimado de la variable ST, el cual mostró un signo negativo, reflejo de que son sustituibles; sin embargo, el valor estimado de dicho parámetro no resultó ser significativamente diferente de cero, por lo tanto rechazamos dicha hipótesis nula. Como resultado de los valores que se han encontrado de los parámetros de este modelo se puede establecer, de acuerdo con la ecuación i), que la expresión:

$$
\text { ii) } r_{s}=0.0096 S_{i}
$$

nos indica la relación entre las tasas marginales de retorno y el nivel de escolaridad.

En lo que respecta a la variable experiencia, la formulación del modelo permite probar la cuarta hipótesis, la cual establece que los ingresos derivados del trabajo siguen el patrón propuesto por la hipótesis del ciclo de vida; es decir, que crecen con la experiencia hasta llegar a un máximo, después del cual descienden.

Observando los valores estimados de los coeficientes $T$ y $T^{2}$, se puede notar que éstos difieren significativamente de cero y tienen los signos planteados por dichas hipótesis; por lo tanto aceptamos ésta con un nivel de confianza del 95 por ciento.

Considerando el valor estimado de los coeficientes para cada uno de los trimestres, se pueden hacer cálculos con base en algunos de ellos para determinar el número de años de experiencia $\left(T^{*}\right)$ en el cual se llega a un máximo de ingresos del trabajo; al realizar esto se encuentra que $T^{*}=38.5$ años.

Al considerar las estimaciones de los parámetros de este modelo para el grupo de hombres y mujeres (cuadro 4), se encontró, mediante la utilización de las pruebas $F$, que los conjuntos de coeficientes tenían diferencia significativa. Analizando las estimaciones correspondientes al grupo masculino, se observaron resultados semejantes a los descritos en los tres párrafos anteriores. Un dato interesante se presenta en el grupo femenino, en donde en términos generales, se puede decir que los coeficientes de las variables $S T, S$ y $S^{2}$ no difieren significativamente de cero, en tanto que los relacionados con las variables $T$ y $T^{2}$ sí lo hacen, lo que refleja que el cambio en la especificación del modelo hace significativo el efecto de la experiencia sobre el ingreso del trabajo, en tanto que origina que los rendimientos estimados de la escolaridad no difieran significativamente de cero.

Buscando introducir a este modelo las mismas modificaciones que al primero, se incluyó la variable horas trabajadas por semana $(H T)$, de donde surge por lo tanto un quinto modelo:

E. Modelo V. Escolaridad, experiencia, horas trabajadas y ocupación

17 Véase p. 14. 


$$
\text { v) } \begin{aligned}
1_{n} Y_{i}= & \alpha_{0}+\alpha_{1} s_{i}+\alpha_{2} S_{i}^{2}+\alpha_{3} T_{i}+\alpha_{4} T_{i}^{2}+\alpha_{5} H T_{i}+ \\
& \alpha_{6} D_{2 i}+U_{i}
\end{aligned}
$$

Analizando las estimaciones de los parámetros de este modelo (cuadro 5), se encuentra, para el caso general que el coeficiente $H T$ tiene valor positivo y significativamente diferente de cero, y lo que es más importante, que ante la introducción de $H T$ no se producen variaciones significativas en los valores de los coeficientes $S, S^{2}, T$ y $T^{2}$. Este mismo patrón se observa cuando las regresiones se obtienen para los grupos masculino y femenino (cuadro 5).

Por otra parte, cuando se incorporan en el modelo los sectores de la actividad económica:

F. Modelo VI: Escolaridad, experiencia, sector de actividad económica (función cuadrática)

VI. $1_{n} Y_{i}=\alpha_{0}+\alpha_{1} S_{i}+\alpha_{2} T_{i}+\alpha_{3} D_{2 i}+\alpha_{4} D_{4 i}+\ldots+$

$$
\alpha_{13} D_{13 i}+\alpha_{14} T_{i}^{2}+\alpha_{15} S_{i}^{2}+U_{i}
$$

Para la estimación de las tasas marginales de rendimiento a la escolaridad (modelo VI), se encuentran resultados semejantes a los del modelo anterior, es decir, que en términos generales los coeficientes de las variables $S, S^{2}, T$ y $T^{2}$ no difieren significativamente de las estimaciones del modelo (IV); este tipo de comportamiento se presenta tanto para las estimaciones del grupo masculino como para las del femenino. Por lo tanto, como resultado de las estimaciones provenientes del modelo (IV) se obtienen tasas marginales de rendimiento a la educación presentadas en el cuadro 2.

De esta manera, por medio de los seis modelos sobre los ingresos provenientes del trabajo, se han probado seis hipótesis que muestran las relaciones entre ingreso, por una parte, y escolaridad y experiencia, por otra, que son propuestas por la teoría del capital humano. La evidencia muestra que el retorno marginal social a la escolaridad es significativamente positivo, $e$ independiente del sexo, experiencia o sector de la actividad económica; implica por lo tanto la importancia de la escolaridad en la política sobre el crecimiento y la distribución del ingreso.

\section{RESUMEN Y CONCLUSIONES}

El estudio parte de dos ideas fundamentales: la primera establece que la escolaridad y la experiencia son formas de adquirir capacidades que, al ser atribuidas por el mercado, permiten incrementar el flujo futuro de ingresos del trabajo generados por el individuo; la segunda plantea que este proceso de acumulación lleva consigo la dedicación de tiempo y recursos que tienen un costo de oportunidad positivo, y que, por lo tanto, no se dan gratis para la sociedad. 


\section{Cuadro 2}

Tasas marginales de retorno a la educación en el área metropolitana de Monterrey

A partir de estas ideas surge la concepción del ser humano como un bien de capital, a la vez que, guiados por el principio de eficiencia en la asignación del gasto público, la preocupación por el menosprecio que queda recibir este factor como fuente de crecimiento económico. Ante estos problemas, el presente estudio explora la evidencia empírica sobre algunas hipótesis que surgen de la concepción mencionada, encontrándose los siguientes resultados según información del mercado de trabajo del área metropolitana de Monterrey 1978:

a) Existe evidencia de una fuerte relación positiva entre la escolaridad y los ingresos provenientes del trabajo, lo cual sugiere que los aumentos en los niveles educativos repercuten sobre el nivel de ingreso y que existe una relación entre la distribución de la población por educación y la distribución por grupos de ingreso.

b) Existe evidencia sobre una fuerte relación entre la experiencia y los ingresos promedio del trabajo lo cual sugiere una fuerte relación entre la edad promedio de la población económicamente activa y los niveles de los ingresos provenientes del trabajo.

c) Los retornos marginales de la educación se incrementan con el nivel de escolaridad, lo cual sugiere una escasez relativa de recursos humanos calificados, que persiste debido a la rapidez con que aumenta la demanda de personas de elevados niveles de escolaridad y por la existencia de distorsiones de los mercados de trabajo y capital que originan que los rendimientos 
privados difieran fuertemente de los sociales (por diferencias en las tasas marginales de impuesto sobre la renta).

d) El perfil de ingreso de la población económicamente activa se conforma con el patrón señalado por el ciclo de vida, lo que explica una relación estrecha entre la estructura de edades de la población económicamente activa y la distribución personal del ingreso proveniente del trabajo.

e) Existe evidencia de discriminación por sexo en el mercado de trabajo; sin embargo, lo que es más importante, ésta no influye sobre las tasas marginales y medias a la educación.

f) No existe evidencia significativa de que las capacidades adquiridas por la escolaridad y por la experiencia sean complementarias o sustituibles entre sí; por lo tanto, esta segunda variable no influye sobre los rendimientos sociales obtenidos de la escolaridad.

g) Se obtienen estimaciones del rendimiento a la educación que resultan ser significativamente menores a los obtenidos por Carnoy correspondientes a 1963.

La conclusión más importante de estos resultados se refiere a que existe relación entre la escolaridad y el ingreso proveniente del trabajo con la tasa marginal de retorno, pues con ellos se considera la relación de la política gubernamental en materia educativa sobre los problemas de distribución y de crecimiento del ingreso. Si se siguiese el principio de eficiencia, alentándose las políticas gubernamentales que fomentan la formación de recursos con "altos" niveles de rentabilidad, se incrementaría el crecimiento del país, pero también lo haría la inequidad en la distribución del ingreso. Considero que si la decisión fuera dicotómica, la política debiera orientarse a reducir la inequidad en la distribución del ingreso (fomentando el cumplimiento de niveles básicos de escolaridad). Esta conclusión se deduce de las dificultades obtenidas de defender una relación entre el principio de eficiencia y el logro de un máximo de bienestar social cuando no se tiene una función social de bienestar, y del temor de que, guiados por este principio, se incrementen las desigualdades de oportunidad en la acumulación de capital humano, repercutiendo, por lo tanto, en la distribución futura de la riqueza.

De existir la oportunidad de tomar decisiones, creo que la más adecuada sería aquella en que la actividad del gobierno se dirigiera principalmente a dar igualdad de oportunidad a todos los grupos sociales, lo cual implica llevar a cabo programas de salud, nutrición y crédito a la educación (que suprime las distorsiones existentes en el mercado de capitales) que eliminen las desventajas que se presentan en los grupos de bajos ingresos cuando se enfrentan a los filtros educacionales existentes.

Si se llevaran a cabo estas políticas cada individuo se enfrentaría con las mismas oportunidades de elegir su perfil de ingresos futuros; y al estimular la acumulación de capital humano se podría llegar a una solución caracterizada por mayor crecimiento. Con esta opción, la distribución se haría menos inequitativa, si, no obstante eso, se fomenta el crecimiento, la existencia de diferencias en las tasas marginales del impuesto sobre la renta evitaría que éste fuera muy alto. 


\section{Limitaciones}

Lás limitaciones que se obtienen por medio de la metodología empleada en el presente estudio tienden a sobrestimar las tasas de retorno en consideración, ya que hacen caso omiso de los costos directos implicados de este tipo de educación y se omite la variable habilidad (la cual en otros países tiende a encontrarse relacionada positivamente con la escolaridad).

\section{Cuadros anexos}

\section{Cuadro 1}

Análisis de regresión de los ingresos provenientes del trabajo de la población económicamente activa del área metropolitana de Monterrey:* Modelo I

(Enero-abril 1978)

\begin{tabular}{|c|c|c|c|c|}
\hline Variables Independientes & Ge ner a & 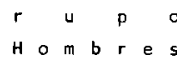 & 5 & eres \\
\hline \multirow[t]{2}{*}{ Constante } & 3.2822 & 3.5229 & & $2.6: 15$ \\
\hline & 15.4629 & $(15.9433)$ & & $(5.3089)$ \\
\hline \multirow[t]{2}{*}{$s$} & .1080 & .1068 & & .1383 \\
\hline & 17.9760 & $(7.9335)$ & & (3.9156: \\
\hline \multirow[t]{2}{*}{02} & 1.9282 & 1.7505 & & 2.2730 \\
\hline & $(11.3742)$ & $(9.7154)$ & & (6. $2344:$ \\
\hline \multirow[t]{2}{*}{$\mathrm{T}$} & .0233 & .0287 & \multicolumn{2}{|r|}{.005} \\
\hline & $(6.4679)$ & $(7.8378)$ & \multicolumn{2}{|r|}{$(.0607)$} \\
\hline \multirow{2}{*}{$\begin{array}{l}\text { No. de Observaciones } \\
R\end{array}$} & 1287 & .951 & \multicolumn{2}{|r|}{336} \\
\hline & .3762 & .4074 & \multicolumn{2}{|r|}{.3789} \\
\hline$R^{2}$ & .1415 & .1660 & \multicolumn{2}{|r|}{.1436} \\
\hline$\Sigma \mathrm{e}^{2}$ & 3567.3394 & 2008,9914 & \multicolumn{2}{|r|}{1377.5105} \\
\hline
\end{tabular}

\footnotetext{
Fuente: Encuesta Continua de Mano de obra

Nota: Coeficientes "t" entre paréntesis: Variable dependiente en têrminos de logaritmos naturales.

* El Area Metropolitana de Monterrey está integrada por los Municipios de Monterrey, Guadalupe, San Nicolás, Santa Catarina, Garza Garcia, Gral. Escobedo y Apodaca.

$*$ No significativos a un nivel de confianza del $95 \%$.
} 


\section{Cuadro 2}

Análisis de regresión de los ingresos provenientes del trabajo de la población económicamente activa del área metropolitana de Monterrey:* Modelo II

(Enero-abril 1978)

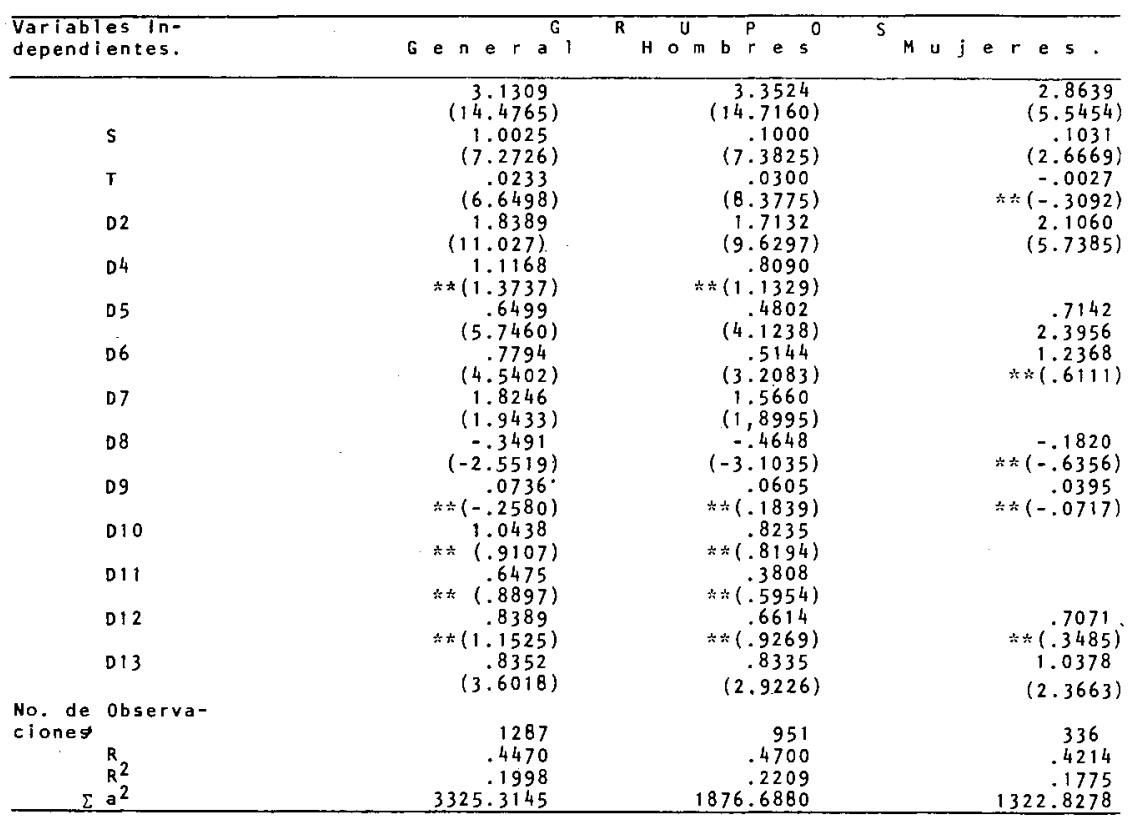

Fuente: Encuesta Continua de Mano de obra.

Nota: Coeficiente "t" entre paréntesis: Variable dependiente en términos de logoris-

- mos naturales.

El Area Metropolitana de Monterrey está integrada por los Municipios de: Monte rrey, Guadalupe, San Nicolás, Santa Catarina, Garza García, Gral. Escobedoy

** No significativos a un nivel de confianza,del $95 \%$. 


\section{Cuadro 3}

Análisis de regresión de los ingresos provenientes del trabajo de la población económicamente activa del área metropolitana de Monterrey:* Modelo III

(Enero-abril 1978)

\begin{tabular}{|c|c|c|c|}
\hline \multirow{2}{*}{$\begin{array}{l}\text { Variables In- } \\
\text { dependientes. }\end{array}$} & \multirow[b]{2}{*}{ General } & $\begin{array}{lllll}R & U & P & 0 & 5\end{array}$ & \multirow[b]{2}{*}{ Mujeres } \\
\hline & & Hombres & \\
\hline Constante & 3.1608 & 3.4116 & 2.7806 \\
\hline$s^{2}$ & $\begin{array}{c}(14.8069) \\
.0995\end{array}$ & $\begin{array}{c}15.19291 \\
.0966\end{array}$ & $\begin{array}{l}13.31901 \\
.1104\end{array}$ \\
\hline$T^{2}$ & $\begin{array}{l}(7.3151) \\
.0222 \\
(6.4083)\end{array}$ & $\begin{array}{l}(7.2320) \\
.0281 \\
(7.9498)\end{array}$ & $\begin{array}{r}(2.8495) \\
-.0009 \\
* \therefore(-.0100)\end{array}$ \\
\hline D2 & $\begin{array}{l}1.2001 \\
6.1085\end{array}$ & $\begin{array}{l}1.0910 \\
5.2391\end{array}$ & $\begin{array}{l}1.6619^{-} \\
(3.7535)\end{array}$ \\
\hline D4 & $\begin{array}{r}1.1299 \\
\therefore *(1.4084\end{array}$ & $\therefore=(1.8384$ & \\
\hline D 5 & $\begin{array}{c}.6279 \\
(5.6232)\end{array}$ & $\begin{array}{c}.4630 \\
(4.0368)\end{array}$ & $\begin{array}{c}.7242 \\
(2.4367)\end{array}$ \\
\hline 06 & $\begin{array}{c}.7354 \\
(4.3367)\end{array}$ & $\begin{array}{c}.4847 \\
(3.0687)\end{array}$ & $=\begin{array}{r}1.1909 \\
*: 5904)\end{array}$ \\
\hline D7 & $\begin{array}{l}(1.8237) \\
(1.9683)\end{array}$ & $\begin{array}{l}1.5880 \\
(1.9563)\end{array}$ & \\
\hline D8 8 & $\begin{array}{c}-.3745 \\
(-2.7724)\end{array}$ & $\begin{array}{r}-.5115 \\
(-3.4626)\end{array}$ & $\therefore *\left(\begin{array}{r}-.1751 \\
-.6135)\end{array}\right.$ \\
\hline D9 & $* *\left\{\begin{array}{r}-.1432 \\
-.5078\end{array}\right.$ & $* *\left(\begin{array}{l}.0454 \\
.1402)\end{array}\right.$ & $\begin{array}{r}.1625 \\
(-.2937)\end{array}$ \\
\hline D 10 & $=\left(\begin{array}{l}.9113 \\
.8057\end{array}\right)$ & $=(.7096)$ & \\
\hline D11 & 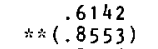 & $=\left(\begin{array}{c}.3607 \\
.5727)\end{array}\right.$ & \\
\hline D 12 & $\therefore *(1.831678)$ & $* \div(.6734)$ & $* *(.6651)$ \\
\hline D 13 & $\begin{array}{c}1.0193 \\
(4.4143)\end{array}$ & $\begin{array}{c}1.0245 \\
(3.6212)\end{array}$ & $\begin{array}{c}1.1202 \\
(2.5485)\end{array}$ \\
\hline HT & $\begin{array}{c}.0160 \\
(5.9491)\end{array}$ & $\begin{array}{c}.0153 \\
(5.5265)\end{array}$ & $\begin{array}{c}.0119 \\
(1.7803)\end{array}$ \\
\hline No de Observa- & & & \\
\hline $\begin{array}{c}\text { ciones. } \\
R_{2} \\
R^{2}\end{array}$ & $\begin{array}{l}1287 \\
.4706 \\
.2214\end{array}$ & $\begin{array}{r}951 \\
.4955 \\
.2455\end{array}$ & $\begin{array}{l}336 \\
.4307 \\
.1855\end{array}$ \\
\hline$\Sigma e^{2}$ & 3235.2960 & 1817.3850 & 1310.0511 \\
\hline
\end{tabular}

Fuente: Encuesta continua de Mano de obra.

Nota: Coeficientes "t" entre paréntesis: Variables dependientes en têrminos de $10-$ garitmos naturales.

* El Area Metropolitana de Monterrey está integrada por los Municipios de Monterrey, Guadalupe, San Nicolás, Santa Catarina, Garza García, Gral. Escobedo

* No significativos a un nivel de confianza del $95 \%$. 


\section{Cuadro 4}

Análisis de regresión de los ingresos provenientes del trabajo de la población económicamente activa del área metropolitana de Monterrey:* Modelo IV

(Enero-abril 1978)

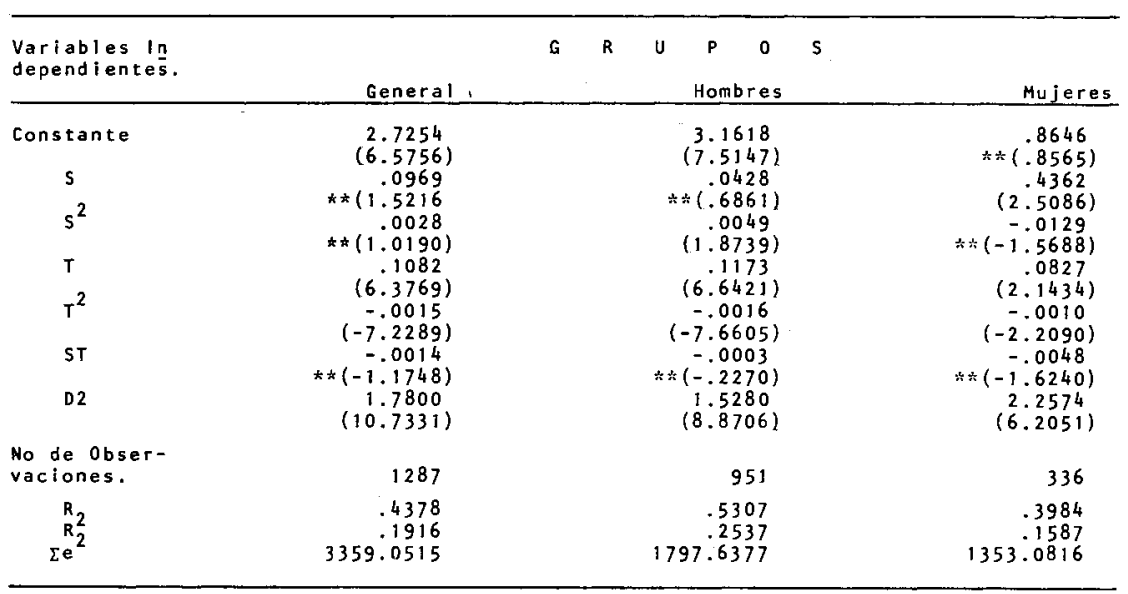

Fuente: Encuesta Continua de Mano de Obra.

Nota: Coeficientes "t" entre parêntesis: Variable dependiente en términos de logarismos naturales.

* El Area Metropolitana de Monterrey está integrada por los Municipios de: Monterrey, Guadalupe, San Nicolás, Santa Catarina, Garza García, Gral. Es

* No significativos a un nivel de confianza del $95 \%$. 


\section{Cuadro 5}

Análisis de regresión de los ingresos provenientes del trabajo de la población económicamente activa del área metropolitana de Monterrey.* Modelo $\mathrm{V}$

(Enero-abril 1978)

\begin{tabular}{|c|c|c|c|c|}
\hline $\begin{array}{l}\text { Variables In- } \\
\text { dependientes }\end{array}$ & G e $n$ e $r$ a 1 & G & 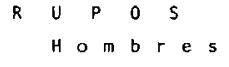 & Mujeres \\
\hline Constante & $\begin{array}{c}3.1610 \\
(12.5773)\end{array}$ & & $\begin{array}{c}3.316 \\
(13.2543)\end{array}$ & $\begin{array}{c}2.0805 \\
(3.2204)\end{array}$ \\
\hline s & $* \div(.0268)$ & & 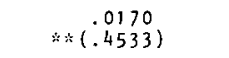 & $\begin{array}{c}.2203 \\
(1.8836)\end{array}$ \\
\hline$s^{2}$ & $\begin{array}{c}.0056 \\
(2.5357)\end{array}$ & & $\begin{array}{c}.0060 \\
(2.9312)\end{array}$ & $\begin{array}{r}-.0043 \\
* \div(.6308)\end{array}$ \\
\hline$T$ & $(10.0882)$ & & $\begin{array}{r}.1092 \\
(12.0512)\end{array}$ & $\approx *(1.03226)$ \\
\hline$T^{2}$ & $\begin{array}{c}-.0013 \\
(-8.6340)\end{array}$ & & $\begin{array}{c}-.0016 \\
(-10.1943)\end{array}$ & $\approx *(-1.6220)$ \\
\hline HT & $\begin{array}{c}.0144 \\
(5.3615)\end{array}$ & & $\begin{array}{c}.0121 \\
(4.4749)\end{array}$ & $\begin{array}{c}.0098 \\
* *(1.4595)\end{array}$ \\
\hline 02 & $\begin{array}{l}1.2048 \\
(6.1798)\end{array}$ & & $\begin{array}{c}1.0394 \\
(5.1453)\end{array}$ & $\begin{array}{c}1.8780 \\
(4.2582)\end{array}$ \\
\hline $\begin{array}{l}\text { No. de Observa- } \\
\text { cianes. } \\
F_{2} \\
R_{2}^{2} \\
\sum \mathrm{e}^{2}\end{array}$ & $\begin{array}{r}1287 \\
.4567 \\
.2086 \\
3288.8165\end{array}$ & & $\begin{array}{r}951 \\
.5188 \\
.2691 \\
1760.3927\end{array}$ & $\begin{array}{r}336 \\
.3968 \\
.1574 \\
1355.1544\end{array}$ \\
\hline
\end{tabular}

Fuente: Encuesta continua de mano de obra

Nota: Coeficientes " $t$ " entre paréntesis: Variable dependiente en términos de

El logaritmos naturales.

El Area Metropolitana de Monterrey está integrada por los Municipios de Monterrey, Guadalupe, San Nicolás, Santa Catarina, Garza García, Gral. Escobedo y -

* No significativos a un nivel de confianza del $95 \%$ 


\section{Cuadro 6}

Análisis de regresión de los ingresos provenientes del trabajo de la población económicamente activa del área metropolitana de Monterrey.* Modelo VI

(Enero-abril 1978)

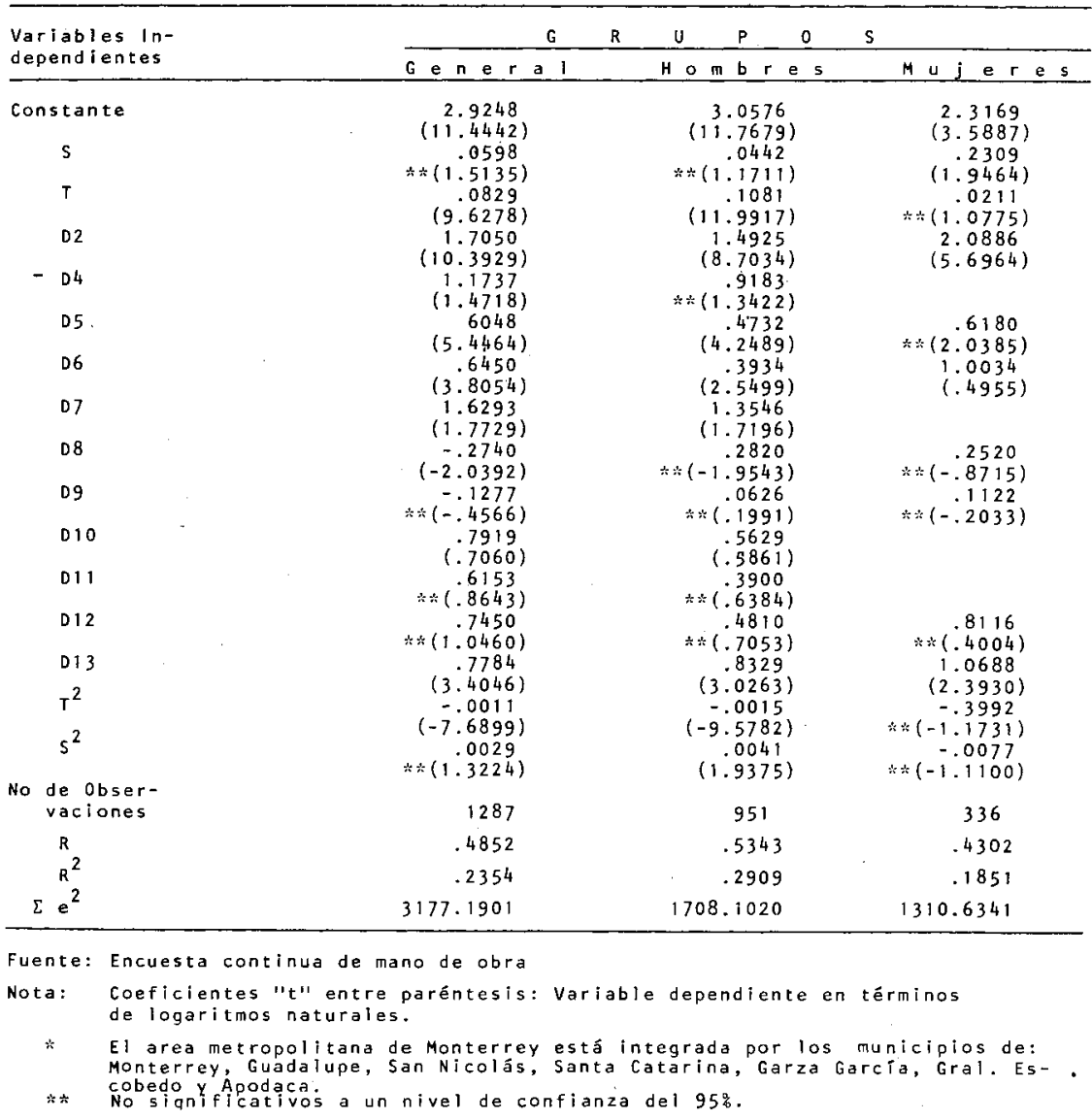




\section{BIBLIOGRAFÍA}

A lan, Thomas. Productivity in Education, University of Chicago Press. Referido por Bowman M.J., op. cit., p. 121.

ANDERSON, C.A. "Diferencias interregionales y raciales relacionadas con los ingresos y la educación". School Review núm. 55 (enero de 1955).

BECKER, GARY. "Economic Theory". Alfred A. Knoff Book in Economics New York, 1971. 1964.

, Human Capital, Columbia University Press, Nueva York y Londres, 1964.

1964. "Investment in Human Capital: A Theorical Analysis". Journal of Political Economy. vol. 70, núm. 5, parte II. Octubre, 1970.

BOWEN, W.G. "Valoración de la contribución económica de la educación". Higher Education, Report of the Committee under the chairmanship of Lord Robbins, 1961-1963. Londres H.M. 1963.

BoWman, MARY J. "Revolución en el pensamiento económico a causa del concepto inversión humana". Sociology of Education. Vol. 39.1966.

Blaug, Mark. Economía de la Educación, Textos Escogidos, Ed. Tecnos. Madrid, 1972.

Carnoy, Martin. "The Cost and Return to Schooling in Mexico: A Case Study". Tesis de doctorado, Universidad de Chicago, 1964.

, "Rates of Return from Schooling in Latin America"/Journal of Human Resources, vol. 2, núm. 1, verano, 1967.

ChIswICK, BAR RY. Income Inequality. Columbia University Press, Nueva York, 1974.

DENISON, E.F. "The Sources of Economic Growth in the United States and the Alternatives Before US". Estudio del Committee for Economic Development, Nueva York, 1962.

- "Measuring the Contribution of Education (and the Residual) to Economic Growth". The Residual in Economic Growth. París: OECD, 1964.

Friedman, Milton. Capitalism and Freedom. The University of Chicago Press, Chicago, 1962.

___- Income from Independent Professional Practice. Nueva York, 1964.

__ Teoria de Precios. Apuntes para un curso en la Universidad de Chicago, Alianza Editorial, Madrid, 1962.

ShafFer, H.G. "Una crítica al concepto de capital humano". American Economic Review. vol. 51, núm. 5, diciembre, 1961.

StINSON ORTIZ, M.H. Yvonne. Los determinantes del ingreso laboral: El enfoque de rendimientos a la escolaridad. Tesis profesional, Universidad Autónoma de Nuevo León, 1979.

HARBINSON y MyERS. Education, Manpower and Economic Growth. McGraw Hill. Nueva York, 1964. 
Hauffman, Wallace. "Decision Marking: The Role of Education". American Journal of Agricultural Economics, febrero, 1974.

Hayami, Yujiro y Ruttan, Vernon W. Agricultural Development: An International Perspective. The John Hopkins Press.

Heckman, James y Polancheck, Solomon. "The Funcional Form of the Income-Schooling Relationship", Journal of the American Statistical Association. Vol. 69, Núm. 346, junio, 1974.

HiRSHLEIFER, JACK. "On the Optimal Theory of Investment Decisions". Journal of Political Economy. Vol. 66, núm. 4, agosto, 1958.

HovarT. "The Optimum Rate of Investment". Economic Joumal. Diciembre, 1958, vol. 68, núm. 272, pp. 747-767. Referido por Schultz.

"Inversión en Capital Humano". American Economic Review. Vol. 51,-núm. 1, marzo, 1961.

JoHNSON, H.G. "Towards a Generalized Capital Accumulation Approach Economic Development". Residual Factors and Economic Growth. OCED, París, 1964.

JohnSton, J. Econometric Methods. McGraw Hill. Nueva York, 1963.

KENDRICK. Productivity Trends in the United States. National Bureau of Economic Research, Princeton University Press for the NBER, 1961. Referido por Bowen, op. cit.

Krueger, ANNe. "Factors Endowments and Per-capita Income Differiencies Among Countries". Economic Joumal, vol. 78, núm. 311, septiembre, 1968.

Solís, Leopoldo. "Mexican Economic Policy in the Post War Period: The Views of Mexican Economist". American Economic Review, vol. 61, núm. 3, suplemento, junio, 1971.

TINBERGEN, JAN Y CORREA, HÉctor. "Quantitative Adaptation of Education to Accelerated Growth. Vol. 12, Kyklos, 1962.

Tinbergen, JAN Y Bos, H.C. "A planning Model for the Education Requirements of Economic Development". The Residual in Economic Growth. OECD, 1965. Referido por Bowman, M.J.op. cit., p. 116.

Grilichrs, ZVi. "Estimating the Returns to Schooling: Some Econometric Problems". Econometrics. Vol. 45, núm. 1, enero, 1977.

- , "Research Expenditure Education and Aggregate Agricultural Production". American Economic Review. Vol. 54, núm. 6, diciembre, 1964.

, Education in Production Function and Growth Accounting in Education, Income and Human Capital. Edited by W. Lee Hansen, noviembre, 1970.

GRILICHES, ZVI Y JORGENSON. "The Sources of Measured Productivity Growth: U.S. Agriculture, 1940-1 960". Journal of Political Economy, vol. 71, núm. 4, agosto, 1963.

Griliches, ZVI Y Mason S. "Education Income and Ability".Journal of Political Economy. Vol. 80, núm. 3, Parte II. Suplemento, mayo-junio, 1972.

Gronau, Rouben. "Age Comparisons Selectivity Bias". Joumal of Political Economy. Vol. 42, núm. 6, noviembre-diciembre, 1934.

LAZER, EDWARD. "Education: Investment of Consumption". Journal of Political Economy. Vol. 85, núm. 3, junio, 1977.

LEWIS, H. GREGG. "Comments on Selectivity Biases in wage Comparisons" Journal of Political Economy. Vol. 82, núm. 6, noviembre-diciembre, 1974. 
LuCAS, Robert W.B. "Hedonic Wage Equations and Psychic Wages in the Returns to Schooling". American Economic Review, vol. 69, núm. 4, septiembre, 1977.

MichaEL, Robert. "The Effect of Education on Efficiency in Consumption". NBER. Nueva York, 1972.

Nicholson, J.S. "The Living Capital of the United Kingdom", Economic Journal. March 1891. Véase J.S. Mill, Principles of Political Economy. (ed.) W.J. Ashley. Londres, 1909.

Schultz, T.W. "Investment in Human Capital: Reply". American Economic Review. Vol. 51, núm. 1, marzo, 1961.

, "The Value of the Ability to Deal with Desequilibrium". Journal of Economic History. Vol. 34, núm. 1, marzo, 1974.

- - Transforming Traditional Agriculture. Arno Press. A New York Times Company, Nueva York, 1976.

, Investment in Education. The University of Chicago Press, 1972.

Secretaría de Programación y Presupuesto: Encuesta continuade mano de obra. Vol. II, núm. 4, septiembre-diciembre 1978, p. 16.

WALSH, Roy. "Capital Concept Applied to Man". The Quarterly Jourmal of Economics. Vol. 69, núm. 2, febrero, 1935.

WEISBROAD. "The Valuation of Human Capital". Journal Political Economics, vol. 69, núm. 5, octubre, 1961. Referido por Schultz, op. cit., pp. 1035-1039.

WELCH, FINIS. "Black and White Rates of Return to Schooling". American Economic Review. Vol. 63, Núm. 5, diciembre, 1973.

- "Education in Production". Journal of Political Economy. Vol. 78, núm. 1, enero-febrero, 1970.

ZEMAn, Morton. "A Quantitative Analysis of White-nonwhite Differentiales in the United States", tesis doctoral sin publicar. Universidad de Chicago, 1955. Referido por Schultz, op. cit., p. 18. 